\title{
Estimated Glomerular Filtration Rate as a Prognostic Factor in Urothelial Carcinoma of the Upper Urinary Tract: A Systematic Review and Meta-Analysis
}

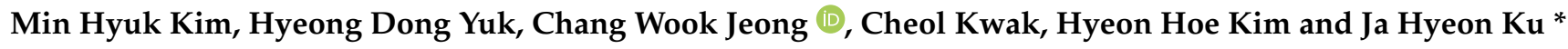

check for

updates

Citation: Kim, M.H.; Yuk, H.D.; Jeong, C.W.; Kwak, C.; Kim, H.H.; Ku, J.H. Estimated Glomerular Filtration Rate as a Prognostic Factor in Urothelial Carcinoma of the Upper Urinary Tract: A Systematic Review and Meta-Analysis. J. Clin. Med. 2021, 10, 4155. https://doi.org/10.3390/ jcm10184155

Academic Editor: Javier C. Angulo

Received: 3 August 2021

Accepted: 14 September 2021

Published: 15 September 2021

Publisher's Note: MDPI stays neutral with regard to jurisdictional claims in published maps and institutional affiliations.

Copyright: (c) 2021 by the authors. Licensee MDPI, Basel, Switzerland. This article is an open access article distributed under the terms and conditions of the Creative Commons Attribution (CC BY) license (https:// creativecommons.org/licenses/by/ $4.0 /)$.
Department of Urology, Seoul National University College of Medicine, Seoul National University Hospital, Seoul 03080, Korea; michael920@hanmail.net (M.H.K.); hinayuk@naver.com (H.D.Y.); drboss@gmail.com (C.W.J.); mdrafael@snu.ac.kr (C.K.); hhkim@snu.ac.kr (H.H.K.)

* Correspondence: kuuro70@snu.ac.kr; Tel.: +82-2-2072-0361

\begin{abstract}
Preoperative renal function is associated with worse outcomes in patients undergoing radical nephroureterectomy (RNU). The purpose of this systemic review and meta-analysis was to determine the association of preoperative renal function with oncological outcome in patients who underwent RNU. We searched articles published up to March 2021 in PubMed, Scopus, and Embase by combining "urothelial carcinoma", "radical nephroureterectomy", and "estimated glomerular filtration rate". We also manually screened the reference list for publications following general guidelines recommended by the preferred reporting items for systematic reviews and meta-analyses (PRISMA) statement. The relationship between preoperative renal function and survival was expressed as overall survival (OS), progression-free survival (PFS), and cancer-specific survival (CSS). This review and meta-analysis included 13 studies involving a total of 4668 patients who received RNU. Pooled analysis showed significant negative association of preoperative renal function with PFS (HR: 1.51, 95\% CI: 1.23-1.80, $p<0.00001$ ), CSS (HR: 1.63, 95\% CI: 1.38-1.92, $p<0.00001$ ), and OS (HR: $1.22,95 \%$ CI: 1.10-1.35, $p<0.00001$ ). Patients with upper tract urothelial carcinoma (UTUC) who received RNU showed a significant negative association of preoperative renal function with survival.
\end{abstract}

Keywords: prognosis; upper urinary tract; urothelial carcinoma; renal function; renal insufficiency

\section{Introduction}

Tumors originating in urothelial cells, including ureter to renal pelvis, are known as upper tract urothelial carcinomas (UTUCs). UTUCs are rare malignant tumors that account for approximately $5-10 \%$ of all urothelial cancers [1-3]. Radical nephroureterectomy (RNU) with bladder cuffing represents the ultimate treatment for highly recurrent UTUC [4,5]. In previous studies, the classification of five-year cancer-specific survival (CSS) was based on pathologic stages. The 5-year CSS exceeded 90\% when the final pathological result was pT1 (non-muscle invasive cancer) or less. However, the 5-year CSS decreased to $40 \%$ when the pathological result was pT3 or higher [6]. This finding suggests that RNU is sufficient for organ-confined early-stage UTUC, whereas RNU alone is insufficient for non-organ confined advanced UTUC with non-organ confined or lymph node metastasis. Complete lymph node dissection along with RNU can increase CSS in patients with UTUCs (pT3 or higher) [7-10]. Other studies reported that adjuvant chemotherapy with locally advanced UTUC (pT3N0/Nx, pT4N0/Nx, or pTanyN+) can effectively increase CSS [11]. Based on the findings of previous studies, locally advanced UTUCs are an indication for lymph node dissection and adjuvant chemotherapy.

Several prognostic factors for UTUC have been reported. Postoperative pathological parameters such as pathologic tumor stage (pT), lympho-vascular invasion (LVI), tumor grade, tumor necrosis, lymph node (LN) involvement, surgical margin, and histological variants are strong prognostic factors. Preoperative prognostic factors include the presence 
of hydronephrosis, serum CRP, tumor size, tumor location, history of previous bladder cancer, age, Eastern Cooperative Oncology Group performance status (ECOG PS), and chronic kidney disease (CKD) [12-21]. However, most of the studies reporting various prognostic factors were retrospective in design.

CKD is a common disease diagnosed in the elderly population. It is associated with malignancies of kidney and ureter [22,23]. The underlying treatment for UTUC is radical resection of the kidney, leaving the patient with a unilateral kidney for survival after surgery. As the unilateral kidney after the surgery needs to perform the function of both kidneys, the patient's renal function might be reduced compared with the level before the surgery, which could result in CKD and affect survival [24,25] Renal function is particularly important in patients with locally advanced UTUC because of the need for adjuvant chemotherapy after surgery. Although many studies have shown that CKD is a significant preoperative prognostic factor in UTUC, most of these studies are limited by their retrospective format. Therefore, the objective of this study was to analyze the association of preoperative renal function with postoperative survival using a systematic review and meta-analysis.

\section{Materials and Methods}

This systematic review and meta-analysis was conducted and reported in accordance with the general guidelines recommended by the preferred reporting items for systematic reviews and meta-analyses (PRISMA) statement [26]. We reviewed studies involving participants who underwent radical nephrectomy for UTUC, including open laparoscopic and robotic surgical interventions.

\subsection{Data Sources and Search Strategy}

The PRISMA flow chart is shown in Figure 1. We searched PubMed, Scopus, and Embase for related articles published before March 2021. The following search terms and their combinations were used: "urothelial carcinoma", "radical nephroureterectomy", and "estimated glomerular filtration rate". We also manually screened the reference lists of publications to identify potentially relevant studies for analysis.

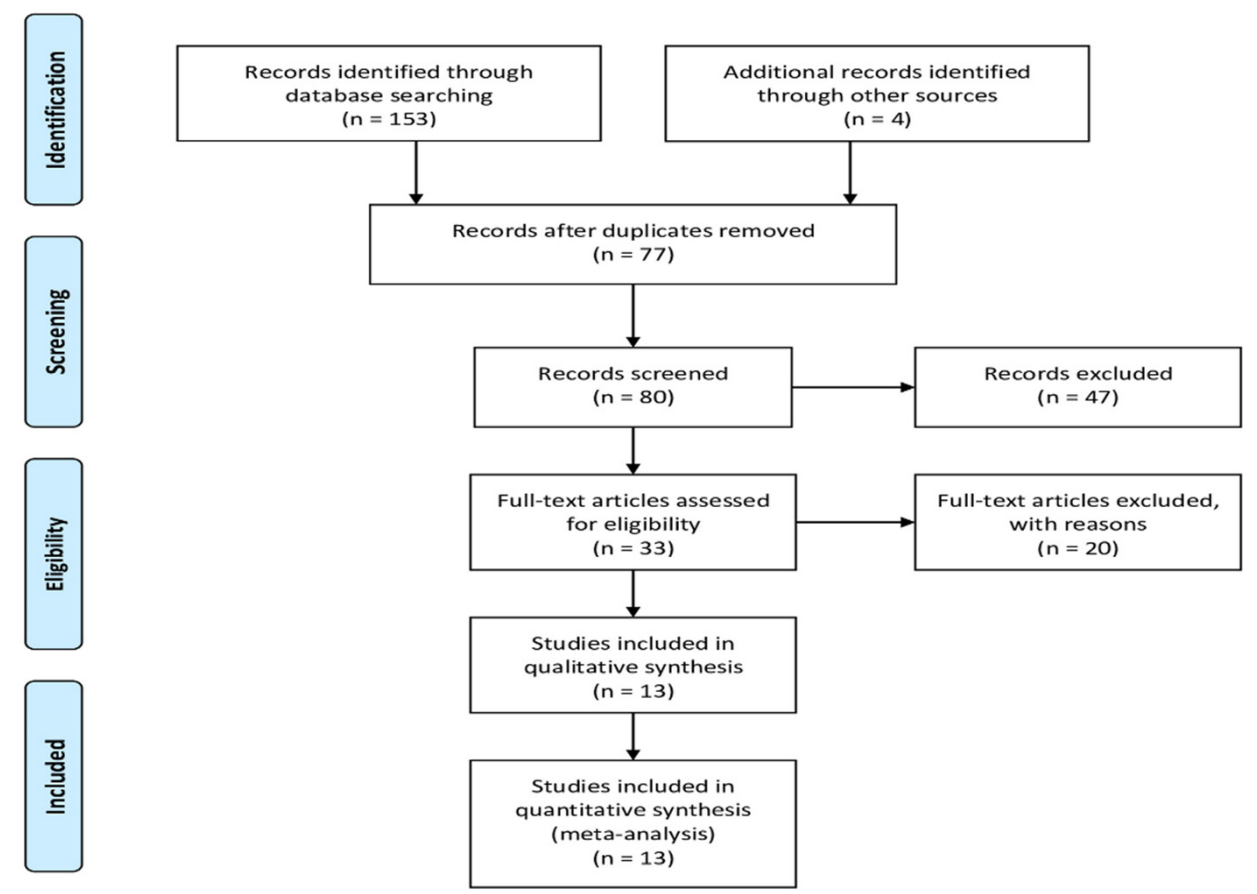

Figure 1. A PRISMA flowchart of the literature search strategy used in our meta-analysis and systemic review. 


\subsection{Inclusion and Exclusion Criteria}

We used strict inclusion and exclusion criteria to limit the heterogeneity of the entire study. If the study met the following including criteria, it was eligible for additional evaluation: (1) patients diagnosed with urothelial carcinoma via pathological testing; (2) determination of relationship between preoperative renal function and prognosis; and (3) studies describing the hazard ratio (HR) and 95\% confidence interval (CI) using multivariate survival analysis.

The exclusion criteria were: (1) letters, comments, case reports, reviews, and conference abstracts with limited data; (2) publications in languages other than English; (3) studies performed using animals or cell lines; and (4) duplicate articles and articles with duplicate data. If the same patient population was evaluated in several studies, only the latest or the largest study was included in the analysis. Studies that did not report adjusted HR using multivariate analysis were excluded because of uncertain accuracy of HR without multivariate analysis. For studies that leveraged both multivariate and univariate analyses to estimate clinical outcomes, results of the multivariate analysis were used to calculate HR and CI. Each study was independently screened by two reviewers (M.H.K. and J.H.K.) to determine compliance with the selection criteria. Any disagreement was resolved by consensus. Finally, 13 papers $[7,11,22,27-36]$ were included in this study.

\subsection{Data Extraction}

Two investigators (H.D.Y. and C.W.J.) reviewed each eligible article individually and extracted information from all publications that met the inclusion criteria. Information was retrieved based on the Reporting Recommendations for Tumor Marker Prognostic Studies (REMARK) involving prognostic markers. The data table was configured to extract all relevant data contained in each study text, table, or figure. Disagreement was resolved through discussion.

\subsection{Quality Assessments}

Methodological quality of each study was evaluated independently by three reviewers (M.H.K., C.K. and H.H.K.) using the Newcastle-Ottawa scale (NOS) including cohort studies only (Supplementary Materials Table S1). A maximum score of 1 was assigned to each item and a maximum score of 2 was allowed only for comparability. Therefore, the final quality score varied from 0 (the lowest) to 9 (the highest), and a total score of $0-5$ was considered low, 6-7 was considered intermediate, and 8-9 was considered high quality.

\subsection{Definition of Survival}

Definition 1. Survival is defined as follows. Cancer-specific survival (CSS) is the period of survival until death from cancer after surgery, and progression-free survival (PFS) is the period of recurrence of cancer at the surgical site, metastasis to nearby lymph nodes, and metastasis to distant sites. Overall survival (OS) represents the survival period after surgery.

\subsection{Statistical Analysis}

Survival data were compiled based on the time-to-event occurrence HR for operating measurements. The HRs and 95\% CIs were calculated using a random-effects model. Forest plots were used to estimate the effect of eGFR on patient survival and disease progression. A statistical test of heterogeneity was performed based on the Q-test and I2 test to assess heterogeneity during the study [37]. A $p$-value $>0.05$ and an $\mathrm{I} 2<50 \%$ were considered non-heterogeneous. Potential publication bias was assessed through visual inspection of the funnel plot. Statistical significance was defined at the 0.05 level. All statistical analyses were performed using RevMan 5.4.1 software (Cochrane Collaboration, Cochranehagen, London, UK). 


\section{Results}

Individual characteristics of 13 studies are presented in Table 1. The recruitment period was from 1991 to 2017. The number of patients included in these studies ranged between 70 and 666, with a total of 4668 patients. All 13 were retrospective studies. Of them, 11, 2, and 11 studies mentioned inclusion/exclusion criteria, definition of survival, and definition of eGFR, respectively.

Table 1. Main characteristics of the eligible studies.

\begin{tabular}{cccccccccc}
\hline Study & Year & Country & $\begin{array}{c}\text { Recruitment } \\
\text { Period }\end{array}$ & $\begin{array}{c}\text { No. of } \\
\text { Patients }\end{array}$ & $\begin{array}{c}\text { Prospective } \\
\text { Data } \\
\text { Collection }\end{array}$ & $\begin{array}{c}\text { Inclusion/ } \\
\text { Exclusion } \\
\text { Criteria }\end{array}$ & $\begin{array}{c}\text { Consecutive } \\
\text { Patients }\end{array}$ & $\begin{array}{c}\text { Definition } \\
\text { of } \\
\text { Survival }\end{array}$ & $\begin{array}{c}\text { Definition } \\
\text { of eGFR }\end{array}$ \\
\hline Xylinas & 2013 & Multination & $1994-2007$ & 666 & No & Yes & NA & Yes & Yes \\
Ito & 2014 & Japan & $1999-2012$ & 70 & No & Yes & NA & No & Yes \\
Raman & 2014 & Multination & $2003-2012$ & 414 & No & No & NA & No & Yes \\
Morizane & 2015 & Japan & $2000-2012$ & 345 & No & Yes & NA & Yes & No \\
Yeh & 2015 & Taiwan & $1991-2013$ & 472 & No & Yes & NA & No & Yes \\
Huang & 2016 & Taiwan & $2001-2016$ & 198 & No & Yes & NA & No & Yes \\
Xing & 2016 & China & $2000-2013$ & 192 & No & Yes & NA & No & Yes \\
Yu & 2017 & Korea & $2004-2014$ & 566 & No & Yes & NA & No & Yes \\
Koguchi & 2018 & Japan & $1990-2015$ & 433 & No & Yes & NA & No & Yes \\
Freifeld & 2019 & USA & $1993-2016$ & 245 & No & Yes & NA & No & No \\
Jan & 2019 & Taiwan & $2007-2017$ & 424 & No & Yes & NA & No & Yes \\
Kuroda & 2019 & Japan & $1999-2017$ & 187 & No & No & NA & No & Yes \\
Momota & 2019 & Japan & $1995-2017$ & 456 & No & Yes & NA & No & Yes \\
\hline
\end{tabular}

eGFR: estimated glomerular filtration rate; NA: not available.

Table 2 shows patient characteristics reported in each study. Median age ranged from 67 to 74 years. Three studies did not indicate age. Nine studies described a surgical approach. An open approach was used for 2031 cases and a laparoscopic approach was adopted for 2393 cases. The median follow-up period ranged from 16 to 65 months.

Table 2. Patient characteristics of the eligible studies.

\begin{tabular}{|c|c|c|c|c|c|c|c|}
\hline Study & $\begin{array}{l}\text { Median Age, } \\
\text { Range (Years) }\end{array}$ & $\begin{array}{c}\text { Gender } \\
\text { (Male/Female) }\end{array}$ & $\begin{array}{l}\text { Median BMI, } \\
\text { Range }\left(\mathrm{kg} / \mathrm{m}^{2}\right)\end{array}$ & $\begin{array}{c}\text { ECOG } \\
\text { Performance } \\
\text { Status } \\
(0 / 1 / 2 / 3)\end{array}$ & Smoking & $\begin{array}{l}\text { Surgical Approach } \\
\text { (Open/Laparoscopic) }\end{array}$ & $\begin{array}{c}\text { Median } \\
\text { Follow-Up, } \\
\text { Range } \\
\text { (Months) }\end{array}$ \\
\hline Xylinas & $69.6,54-76$ & $441 / 225$ & $\begin{array}{c}28.2,24-32 \\
\text { (IQR) }\end{array}$ & $445 / 221(1-3)$ & NA & $519 / 147$ & $\begin{array}{c}45.5,24-67 \\
\text { (IQR) }\end{array}$ \\
\hline Ito & NA & $47 / 23$ & NA & NA & NA & $49 / 21$ & $29.2,1-157$ \\
\hline Raman & $70,27-96$ & $257 / 157$ & NA & $82 / 165 / 159 / 8$ & NA & NA & $16,2-120$ \\
\hline Morizane & $74,38-95$ & $234 / 111$ & $22.1,13-34.2$ & $241 / 103(1-3)$ & 175 & 244/101 & $39.9,6.1-160$ \\
\hline Yeh & $67,24-95$ & $204 / 268$ & NA & NA & 99 & $269 / 203$ & $33,1-233$ \\
\hline Huang & $68.6,23.6-91.6$ & $103 / 95$ & NA & NA & 26 & NA & $29.1,6.4-164.9$ \\
\hline Xing & NA & $78 / 114$ & NA & NA & NA & $84 / 145$ & 65, 3-144 \\
\hline$Y u$ & $72,65-76(\mathrm{IQR})$ & $165 / 401$ & NA & $399 / 148 / 20 / 1$ & NA & $142 / 424$ & $31.1,16.2-55.7$ \\
\hline Koguchi & 69, 62-75 (IQR) & $313 / 120$ & NA & NA & 138 & $243 / 190$ & $\begin{array}{c}35.4,13.8-74.5 \\
\text { (IQR) }\end{array}$ \\
\hline Freifeld & 70 (mean) & $152 / 93$ & 29 (mean) & $126 / 98(1-3)$ & NA & NA & 27 \\
\hline Jan & $70,29-96$ & $189 / 235$ & NA & NA & 49 & NA & $35,14-60$ (IQR) \\
\hline Kuroda & $71,38-90$ & $138 / 49$ & NA & NA & NA & $104 / 83$ & $49.2,3.4-209.2$ \\
\hline Momota & NA & $309 / 147$ & NA & $446(0-1) / 10(2-3)$ & NA & $377 / 79$ & 40 \\
\hline
\end{tabular}

BMI: body mass index; ECOG: Eastern Cooperative Oncology Group; IQR: interquartile range; NA: not available.

Tumor and pathologic characteristics are listed in Tables 3 and 4, respectively. Ten studies reported tumor location. The renal pelvis harbored 1830 tumors and the ureter carried 1783 tumors. In nine studies, a total of 656 people received adjuvant chemotherapy. In 11 studies, tumor grade was low in $1335(31 \%)$ cases and high in $2950(69 \%)$ cases. The 
pathologic $\mathrm{T}$ stage was described in nine studies, including $2381(60 \%)$ below pathologic T2 and $1577(40 \%)$ higher than T3.

Table 3. Tumor characteristics of the eligible studies.

\begin{tabular}{|c|c|c|c|c|c|c|}
\hline Study & $\begin{array}{c}\text { History of } \\
\text { Bladder Cancer }\end{array}$ & Hydronephrosis & Tumor Size & $\begin{array}{c}\text { Tumor } \\
\text { Location } \\
\text { (Pelvis/Ureter) }\end{array}$ & $\begin{array}{c}\text { Tumor } \\
\text { Multifocality }\end{array}$ & $\begin{array}{c}\text { Adjuvant } \\
\text { Chemotherapy }\end{array}$ \\
\hline Xylinas & 244 & NA & NA & $420 / 246$ & 164 & 62 \\
\hline Ito & 17 & 26 & NA & $0 / 70$ & 7 & 23 \\
\hline Raman & NA & NA & NA & NA & NA & 55 \\
\hline Morizane & 36 & 201 & NA & $140 / 205$ & 51 & NA \\
\hline Yeh & NA & NA & NA & $189 / 193$ & 90 & 87 \\
\hline Huang & 49 & NA & NA & NA & NA & 21 \\
\hline Xing & NA & 119 & NA & $102 / 90$ & 52 & NA \\
\hline $\mathrm{Yu}$ & 111 & 249 & NA & $258 / 308$ & 49 & 205 \\
\hline Koguchi & NA & NA & NA & $239 / 194$ & NA & 99 \\
\hline Freifeld & 80 & 71 & 3.4 (mean) & $116 / 85$ & 35 & NA \\
\hline Jan & 127 & 344 & NA & $191 / 138$ & 116 & 40 \\
\hline Kuroda & 47 & 112 & NA & NA & NA & NA \\
\hline Momota & NA & 288 & NA & $175 / 254$ & NA & 64 \\
\hline
\end{tabular}

NA: not available.

Table 4. Pathologic characteristics of the eligible studies.

\begin{tabular}{|c|c|c|c|c|c|c|c|}
\hline Study & $\begin{array}{c}\text { Tumor } \\
\text { Grade } \\
\text { (Low/High) }\end{array}$ & $\begin{array}{l}\text { Pathologic T Stage } \\
\text { (pT0/Is/a/1/2/3/4) }\end{array}$ & $\begin{array}{l}\text { Pathologic } \\
\text { N Stage } \\
(\mathrm{pNx} /-/+)\end{array}$ & $\begin{array}{l}\text { Variant } \\
\text { Form }\end{array}$ & LVI & $\begin{array}{c}\text { Concomitant } \\
\text { CIS }\end{array}$ & $\begin{array}{c}\text { Positive } \\
\text { Surgical } \\
\text { Margin }\end{array}$ \\
\hline Xylinas & $121 / 533$ & $326(\leq \mathrm{T} 1) / 118 / 182 / 40$ & $291 / 291 / 84$ & NA & 171 & 229 & NA \\
\hline Ito & NA & NA & NA & NA & NA & NA & NA \\
\hline Raman & $116 / 298$ & $3 / 16 / 106 / 60 / 60 / 143 / 26$ & $165 / 203 / 46$ & NA & NA & NA & 25 \\
\hline Morizane & $222 / 109$ & $188(\leq \mathrm{T} 2) / 152(\geq \mathrm{T} 3)$ & $205 / 119 / 21$ & 29 & 102 & 43 & 22 \\
\hline Yeh & $112 / 360$ & $0 / 60$ (Tis/a)/130/112/142/28 & $261 / 170 / 41$ & 8 & NA & NA & NA \\
\hline Huang & $11 / 147$ & $198(\mathrm{~T} 3)$ & 198 (N0) & NA & 31 & 20 & 5 \\
\hline Xing & $170 / 22$ & $30(\mathrm{Ta}) / 162(\mathrm{~T} 1)$ & 192 (N0) & NA & NA & NA & NA \\
\hline$Y u$ & $182 / 388$ & $\begin{array}{c}0 / 84 \\
\text { (Tis/a)/128/134/200/20 }\end{array}$ & NA & NA & 119 & 54 & NA \\
\hline Koguchi & $300 / 127$ & $0 / 18 / 66 / 91 / 81 / 153 / 24$ & $181 / 221 / 31$ & NA & 151 & NA & 30 \\
\hline Freifeld & NA & NA & NA & NA & NA & NA & NA \\
\hline Jan & $22 / 402$ & $\begin{array}{c}0 / 0 / 161(\mathrm{Ta} / 1) / 83 / 180 \\
(\mathrm{~T} 3 / 4)\end{array}$ & $\begin{array}{c}399 \\
(\mathrm{Nx} /-) / 25\end{array}$ & NA & 115 & NA & NA \\
\hline Kuroda & $55 / 132$ & $96(\leq \mathrm{T} 2) / 91(\geq \mathrm{T} 3)$ & $0 / 172 / 15$ & NA & 65 & 22 & 21 \\
\hline Momota & $24 / 432$ & $260(\leq \mathrm{T} 2) / 196(\geq \mathrm{T} 3)$ & $\begin{array}{c}431 \\
(\mathrm{Nx} /-) / 25\end{array}$ & NA & 160 & NA & 15 \\
\hline
\end{tabular}

LVI: lymphovascular invasion; CIS: carcinoma in situ; NA: not available.

Table 5 presents results of multivariate analyses using the Cox proportional hazards model. Survival analysis was expressed as overall survival (OS), progressionfree survival (PFS), or cancer-specific survival (CSS). The standard of eGFR was set at $30 \mathrm{~mL} / \mathrm{min} / 1.73 \mathrm{~m}^{2}$ in one study, $50 \mathrm{~mL} / \mathrm{min} / 1.73 \mathrm{~m}^{2}$ in two studies, and $60 \mathrm{~mL} / \mathrm{min} / 1.73 \mathrm{~m}^{2}$ in the remaining studies. 
Table 5. Estimation of hazard ratios.

\begin{tabular}{|c|c|c|c|c|}
\hline Study & $\begin{array}{l}\text { Survival } \\
\text { Analysis }\end{array}$ & $\begin{array}{l}\text { Threshold of eGFR } \\
\left(\mathrm{mL} / \mathrm{min} / 1.73 \mathrm{~m}^{2}\right)\end{array}$ & Co-Factors & Analysis Results \\
\hline Xylinas & OS & 60 & \multirow{2}{*}{$\begin{array}{c}\text { Standard clinico-pathological features } \\
\text { cT stage (T3), length of cancer }(3 \mathrm{~cm}) \text {, maximal } \\
\text { diameter of cancer }(1.6 \mathrm{~cm}), \text { NLR }(3)\end{array}$} & Not significant \\
\hline Ito & PFS & 60 & & Significant \\
\hline Raman & CSS & 60 & $\begin{array}{l}\text { Gender, race, age (70 years), ECOG performance } \\
\text { status }(0,1 / 2,3), \text { pT stage (T3/T4), LN status, } \\
\text { surgical margin status, adjuvant chemotherapy }\end{array}$ & Not significant \\
\hline Morizane & CSS & 50 & $\begin{array}{c}\text { ECOG performance status }(0 / \geq 1) \text {, number of } \\
\text { tumor }(1 />1), \operatorname{CRP}(0.5)\end{array}$ & Significant \\
\hline Yeh & CSS/OS & 60 & $\begin{array}{l}\text { Gender, age (67 years), smoking, surgery method } \\
\text { (laparoscopic/open), tumor location, pT stage, } \\
\text { pN stage, tumor grade, adjuvant chemotherapy, } \\
\text { hematuria, hydronephrosis and flank pain }\end{array}$ & Significant/Significant \\
\hline Huang & $\mathrm{PFS} / \mathrm{CSS} / \mathrm{OS}$ & 60 & $\begin{array}{c}\text { Gender, age (68.6 years), current smoking, ASA } \\
\text { score, recurrent bladder tumor, recurrent } \\
\text { contralateral UTUC, tumor grade, LVI, CIS, } \\
\text { surgical margin status, adjuvant radiotherapy, } \\
\text { adjuvant chemotherapy }\end{array}$ & $\begin{array}{l}\text { Significant/Significant/ } \\
\text { Significant }\end{array}$ \\
\hline Xing & CSS & 30 & $\begin{array}{c}\text { ABCC6 methylation, GDF15 methylation, tumor } \\
\text { multifocality, surgery method } \\
\text { (laparoscopic/open) }\end{array}$ & Significant \\
\hline \multirow[t]{3}{*}{ Yu } & PFS & 60 & BMI, pT stage $(\leq \mathrm{T} 2 / \geq \mathrm{T} 3)$, tumor grade, $\mathrm{LVI}$ & Significant \\
\hline & CSS & 60 & $\begin{array}{c}\mathrm{DM}, \mathrm{pT} \text { stage }(\leq \mathrm{T} 2 / \geq \mathrm{T} 3) \text {, tumor grade, } \mathrm{LVI} \text {, } \\
\text { adjuvant chemotherapy }\end{array}$ & Significant \\
\hline & OS & 60 & $\begin{array}{c}\text { Age, BMI, ECOG performance status }(0,1 / 2,3) \\
\text { tumor size, tumor multifocality, pT stage } \\
(\leq \mathrm{T} 2 / \geq \mathrm{T} 3), \text { tumor grade, } \mathrm{LVI} \text {, adjuvant } \\
\text { chemotherapy }\end{array}$ & Significant \\
\hline Koguchi & PFS/OS & 60 & $\begin{array}{c}\text { Change rate of eGFR, age, gender, tumor location, } \\
\text { tumor grade, } \mathrm{pT} \text { stage, } \mathrm{pN} \text { stage, LVI, surgical } \\
\text { margin status }\end{array}$ & $\begin{array}{l}\text { Not significant/Not } \\
\text { significant }\end{array}$ \\
\hline Freifeld & PFS & 50 & $\begin{array}{c}\text { Age (66 years), ECOG performance status }(0 / \geq 1) \\
\text { hemoglobin, hydronephrosis, } \mathrm{pT} \text { stage } \\
(\leq \mathrm{T} 2 / \geq \mathrm{T} 3), \text { tumor architecture }\end{array}$ & Not significant \\
\hline & & & $\begin{array}{l}\text { Gender, blood type, age ( } 69 \text { years), smoking, } \\
\text { hemodialysis, DM or hypertension, previous or } \\
\text { concomitant bladder cancer, hydronephrosis, }\end{array}$ & \\
\hline Jan & PFS/CSS/OS & 60 & $\begin{array}{l}\text { hematuria, pT stage, pN stage, tumor grade, LVI, } \\
\text { tumor location, tumor multifocality, tumor size } \\
(3 \mathrm{~cm}) \text {, tumor architecture, tumor necrosis, } \\
\text { adjuvant chemotherapy, NLR (4), PLR (150), MLR } \\
(0.4), \text { SII (580) }\end{array}$ & $\begin{array}{l}\text { Not significant/Not } \\
\text { significant/Not significant }\end{array}$ \\
\hline Kuroda & PFS & 60 & $\begin{array}{c}\text { Tumor histology, pT stage }(\leq \mathrm{T} 2 / \geq \mathrm{T} 3) \text {, tumor } \\
\text { grade, pN stage, surgical margin status, LVI, CAR } \\
(0.079, \text { NLR (2.035), PLR (165), GPS (1), fibrinogen } \\
\text { (337) }\end{array}$ & Significant \\
\hline & CSS & 60 & $\begin{array}{c}\text { Urine cytology, tumor histology, pT stage } \\
(\leq \mathrm{T} 2 / \geq \mathrm{T} 3), \text { tumor grade, pN stage, surgical } \\
\text { margin status, LVI, CAR (0.079, NLR (2.035), PLR } \\
\text { (165), GPS (1), fibrinogen (337) }\end{array}$ & Significant \\
\hline Momota & $\mathrm{PFS} / \mathrm{CSS} / \mathrm{OS}$ & 60 & $\begin{array}{c}\text { age, gender, ECOG performance status, } \\
\text { hypertension, CVD, DM, neoadjuvant } \\
\text { chemotherapy, hydronephrosis, } \\
\text { tumour location, tumor grade, pT stage } \\
(\leq \mathrm{T} 2 / \geq \mathrm{T} 3), \mathrm{pN} \text { stage, LVI }\end{array}$ & $\begin{array}{c}\text { Significant/Not } \\
\text { significant/Not significant }\end{array}$ \\
\hline
\end{tabular}

eGFR: estimated glomerular filtration rate; OS: overall survival; PFS: progression-free survival; NLR: neutrophil-to-lymphocyte ratio; CSS: cancer-specific survival; ECOG: Eastern Cooperative Oncology Group; LN: lymph node; CRP: C-reactive protein; ASA: American Society of Anesthesiologists; UTUC: upper tract urothelial carcinoma; LVI: lymphovascular invasion; CIS: carcinoma in situ; BMI: body mass index; DM: diabetes mellitus; PLR: platelet-to-lymphocyte ratio; MLR: monocyte-to-lymphocyte ratio; SII: systemic immune-inflammation index; CAR: C-reactive protein-to-albumin ratio; GPS: Glasgow prognostic score; CVD: cardiovascular disease.

Figure 2 presents a forest plot and a funnel plot to demonstrate PFS according to preoperative renal function. Eight studies were included. Five studies showed significant positive association between PFS and preoperative renal function (adjusted HR: 1.51; 95\% CI: $1.23-1.80, p<0.00001)$. The funnel plot was relatively symmetrical, showing no evidence of significant publication bias. 


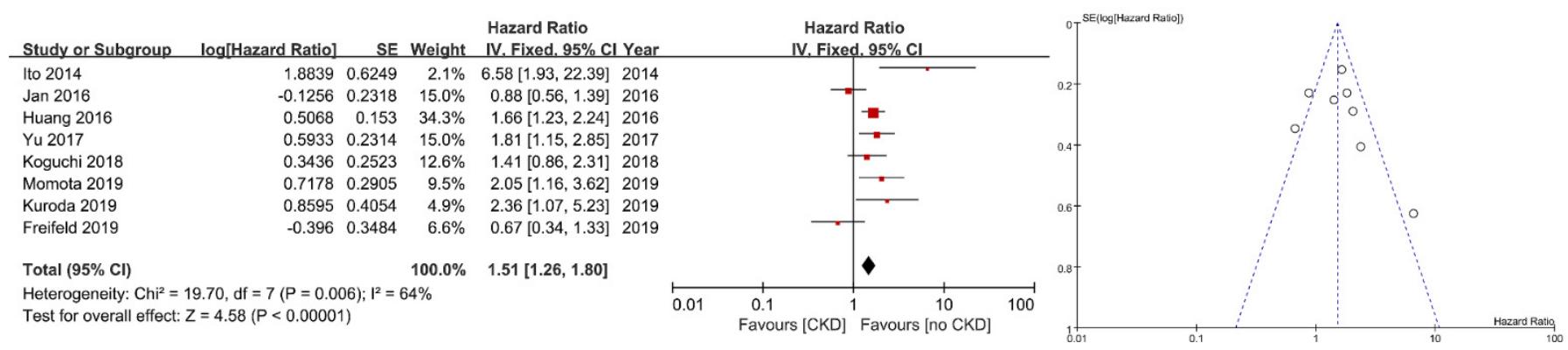

Figure 2. Forest plot and funnel plot of progression-free survival after radical nephroureterectomy according to preope-ative renal function. SE: standard error; IV: inverse variance; CI: confidence interval; df: degree of freedom.

Nine studies showed a relationship between CSS and preoperative renal function. The forest plot and funnel plot are shown in Figure 3. All but one study showed a positive association between CSS and preoperative renal function. Six studies showed a significant positive association between CSS and preoperative renal function (adjusted HR: 1.63, 95\% CI: 1.38-1.92, $p<0.00001$ ). The funnel plot was relatively symmetrical, without strong evidence for publication bias.
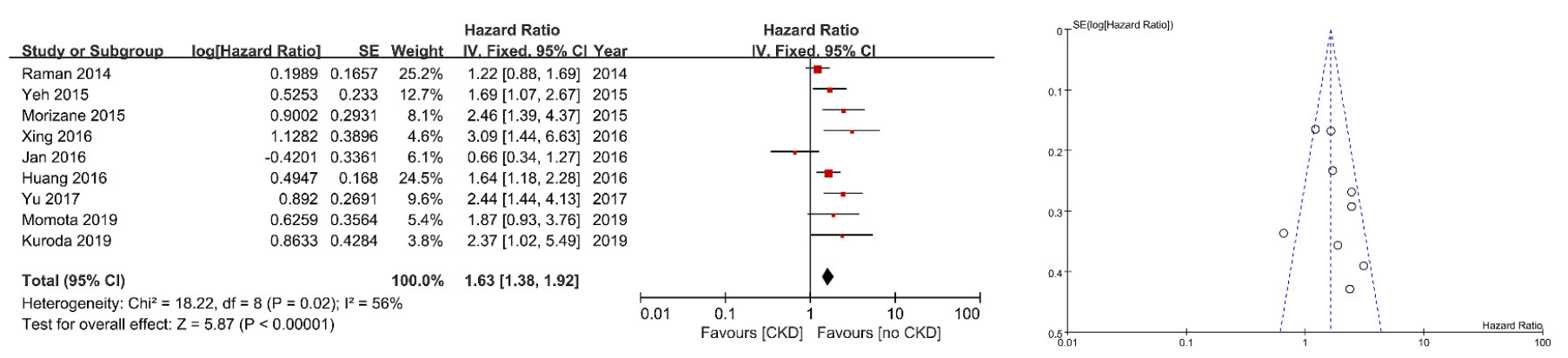

Figure 3. Forest plot and funnel plot of cancer-specific survival after radical nephroureterectomy according to preparative renal function.

Seven studies showed a relationship between OS and preoperative renal function. Forest plots and funnel plots are shown in Figure 4. All but one study showed a positive association between OS and preoperative renal function. Three studies showed a significant positive association between OS and preoperative renal function (adjusted HR: 1.22, 95\% CI: $1.10-1.35, p<0.00001)$. We could not find a strong evidence for publication bias based on the funnel plot.

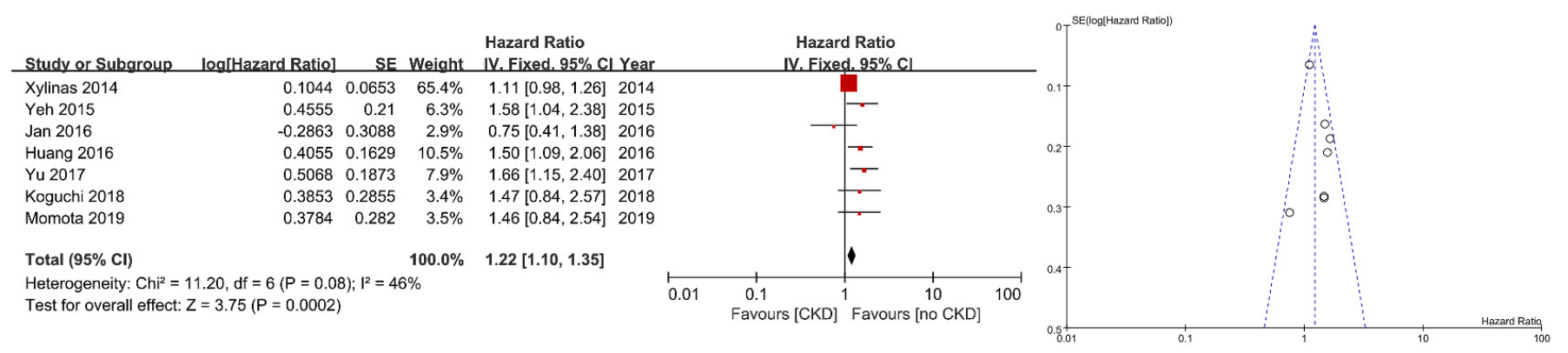

Figure 4. Forest plot and funnel plot of overall survival after radical nephroureterectomy according to preoperative renal function. 


\section{Discussion}

This study investigated whether renal function of patients with UTUC before RNU was associated with survival rate. Thirteen studies were included. The total number of patients was 4668. All patients were diagnosed with UTUC and, therefore, underwent RNU. Each study expressed the survival rate as CSS, PFS, or OS. The final meta-analysis showed that preoperative renal function was related to postoperative survival rate.

Ito et al. [7] analyzed 70 patients with NOM0 UTUC who underwent unilateral RNU between 1999 and 2012. The survival rate was expressed as a 3-year extraurothelial recurrencefree survival rate (EURFS). In the multivariate Cox proportional hazards model, the EURFS had a worse outcome in patients with a preoperative eGFR less than $60 \mathrm{~mL} / \mathrm{min} / 1.73 \mathrm{~m}^{2}$ (HR: 6.579, 95\% CI: 1.934-22.222, $p=0.0026$ ). Yeh et al. [35] investigated the postoperative prognosis according to the presence of preoperative hydronephrosis and flank pain in 472 UTUC patients who underwent RNU in a single medical center from 1991 to 2013. The survival rate was expressed as 5-year CSS and 5-year OS using the Kaplan-Meier method. The eGFR was set at $60 \mathrm{~mL} / \mathrm{min} / 1.73 \mathrm{~m}^{2}$. Those with preoperative hydronephrosis and flank pain had worse outcomes of 5-year CSS and 5-year OS, respectively. Since preoperative hydronephrosis and flank pain were associated with preoperative renal function, patients with eGFR less than $60 \mathrm{~mL} / \mathrm{min} / 1.73 \mathrm{~m}^{2}$ before surgery had worse outcomes of 5-year CSS (HR: 1.691, 95\% CI: 1.071-2.669, $p=0.024$ ) and 5-year OS (HR: 1.577, 95\% CI: 1.045-2.382, $p=0.030$ ). Ehdaie et al. [38] developed a model to predict the prognosis of 253 patients who underwent RNU for UTUC between 1995 and 2008. A multivariable Cox regression model was used and eGFR was set as a continuous variable. Survival rates were expressed as 5-year CSS and 5-year PFS. The higher the preoperative eGFR, the better was the 5-year PFS (HR: 0.73, 95\% CI: 0.61-0.88, $p<0.001$ ) and 5-year CSS (HR: 0.74, 95\% CI: $0.61-0.90, p=0.002)$.

These preceding studies showed that preoperative renal function was positively correlated with CSS, PFS, and OS of patients with UTUC. However, Xylinas et al. [28] showed no association between preoperative renal function and survival rate of patients with UTUC who underwent RNU. Xylinas et al. investigated 781 patients with UTUC treated with RNU from 1994 to 2007 at seven different centers. The preoperative eGFR criterion was set at $60 \mathrm{~mL} / \mathrm{min} / 1.73 \mathrm{~m}^{2}$, and the postoperative eGFR criterion was set at $45 \mathrm{~mL} / \mathrm{min} / 1.73 \mathrm{~m}^{2}$. Univariable and multivariable Cox regression models were used. Neither preoperative nor postoperative eGFR was associated with 5-year CSS, PFS, or OS. In our systematic review and meta-analysis, eight studies demonstrated a relationship between PFS and preoperative eGFR (adjusted HR: 1.51, 95\% CI: 1.23-1.80, $p<0.00001$ ). In five studies, PFS and preoperative eGFR showed a significant positive correlation. Although one study showed a positive correlation and two studies showed a negative correlation, all three studies showed no significant correlations. Nine studies showed a relationship between CSS and preoperative eGFR. Although one study showed a negative correlation between CSS and preoperative eGFR and two studies showed a positive correlation, none of them showed statistically significant correlation between CSS and preoperative eGFR. The remaining six studies showed a significant positive relationship between the two (adjusted HR: 1.63, 95\% CI: $1.38-1.92, p<0.00001)$. Seven studies showed a relationship between OS and preoperative eGFR. Although one study showed a negative relationship, the correlation was not significant. Six studies showed a positive relationship between OS and preoperative eGFR. However, only three studies showed significant correlation between the two variables (adjusted HR: 1.22, 95\% CI, 1.10-1.35, $p<0.00001$ ). Results of this study confirmed that the preoperative renal function of patients was closely related to their survival rate after RNU.

Several previous studies have shown that renal function decreases after kidney surgery $[28,39,40]$. Although patients who underwent radical nephrectomy had severe renal impairment more than those who underwent partial nephrectomy, the rate of CKD was increased postoperatively in patients with partial nephrectomy [41]. In addition, UTUC patients who underwent radical nephrectomy had significantly higher serum creatinine increase and higher rates of ESRD hemodialysis than those of RCC patients (HR: 2.9, 95\% 
CI: $1.88-4.49, p<0.001$ ) [39]. Some studies have shown that patients with CKD or ESRD exhibited a lower survival rate than that of those with normal renal function [24,25]. If UTUC patients manifest reduced preoperative renal function, they carry a high probability of developing CKD or ESRD due to their decreased renal function after radical nephrectomybased RNU. Therefore, it can be inferred that they will have poor outcomes such as disease prognosis and survival rate.

Patients with non-organ-confined or lymph node metastasis undergoing UTUC require adjuvant chemotherapy because it is impossible to perform surgical treatment. Adjuvant chemotherapy for UTUC basically entails a combination of gemcitabine and cisplatin [42,43]. Cisplatin-induced nephrotoxicity is well known [44,45]. When cisplatin is absorbed into renal tubular cells, it can trigger an inflammatory response via multiple signaling pathways, leading to histological damage. Cisplatin also affects renal vessels and causes ischemic damage [46]. Therefore, patients with reduced renal function cannot use cisplatin-based chemotherapy and, thus, exhibit a worse survival rate.

Our systematic review and meta-analysis study has several limitations. First, 10 out of 13 studies involved Asians including five from Japan, three from Taiwan, and one each from Korea and China [7,22,27,29,30,32-36]. The remaining three papers included two from a multinational study and one from the United States [11,28,31]. The number of patients included 3343 Asians, 245 Americans, and 1080 patients from multiple nations. Thus, Asians accounted for at least $71 \%$ of all subjects. Since Asians were included in the study performed in the United States and different nations, it limited the evaluation of other ethnic groups. Second, all 13 papers included in this study were retrospective studies. However, most of these studies had an adequate sample size and a NOS quality score of 6 or higher. Finally, there was heterogeneity between papers. The I2 value indicating heterogeneity exceeded 50\% (PFS, 64\%; CSS, 56\%). Thus, the results of this systematic review and meta-analysis require careful interpretation.

In conclusion, this systematic review and meta-analysis revealed that patients with decreased eGFR before surgery manifested poor PFS, CSS, and OS after RNU. However, all studies included in this meta-analysis were retrospective in nature. Thus, a large-scale prospective study is needed in the future.

Supplementary Materials: The following are available online at https: / www.mdpi.com/article / $10.3390 / \mathrm{jcm} 10184155 / \mathrm{s} 1$, Table S1: Assessment of quality of the non-randomized controlled trials (RCTs) using the Newcastle-Ottawa scale for cohort studies.

Author Contributions: Conceptualization, J.H.K. and M.H.K.; methodology, J.H.K.; software, J.H.K.; validation, H.D.Y. and C.W.J.; formal analysis, J.H.K. and M.H.K.; investigation, J.H.K., M.H.K., H.D.Y. and C.W.J.; resources, J.H.K.; data curation, M.H.K., C.K. and H.H.K.; writing-original draft preparation, M.H.K.; writing-review and editing, M.H.K.; visualization, J.H.K.; supervision, J.H.K. All authors have read and agreed to the published version of the manuscript.

Funding: This study received no external funding.

Institutional Review Board Statement: Ethical review and approval were waived for this study because its use of already available cumulative published data.

Informed Consent Statement: Patient consent was waived because of publicly available accumulative published data.

Data Availability Statement: The data presented in this study are available on request from the corresponding author.

Conflicts of Interest: The authors have no conflict of interest to declare.

\section{References}

1. Munoz, J.J.; Ellison, L.M. Upper tract urothelial neoplasms: Incidence and survival during the last 2 decades. J. Urol. 2000, 164, 1523-1525. [CrossRef]

2. Siegel, R.; Ma, J.; Zou, Z.; Jemal, A. Cancer statistics, 2014. CA Cancer J. Clin. 2014, 64, 9-29. [CrossRef] 
3. Raman, J.D.; Messer, J.; Sielatycki, J.A.; Hollenbeak, C.S. Incidence and survival of patients with carcinoma of the ureter and renal pelvis in the USA, 1973-2005. BJU Int. 2011, 107, 1059-1064. [CrossRef] [PubMed]

4. Rouprêt, M.; Babjuk, M.; Comperat, E.; Zigeuner, R.; Sylvester, R.; Burger, M.; Cowan, N.; Böhle, A.; Van Rhijn, B.W.; Kaasinen, E. European guidelines on upper tract urothelial carcinomas: 2013 update. Eur. Urol. 2013, 63, 1059-1071. [CrossRef] [PubMed]

5. Rouprêt, M.; Zigeuner, R.; Palou, J.; Boehle, A.; Kaasinen, E.; Sylvester, R.; Babjuk, M.; Oosterlinck, W. European guidelines for the diagnosis and management of upper urinary tract urothelial cell carcinomas: 2011 update. Eur. Urol. 2011, 59, 584-594. [CrossRef]

6. Hall, M.C.; Womack, S.; Sagalowsky, A.I.; Carmody, T.; Erickstad, M.D.; Roehrborn, C.G. Prognostic factors, recurrence, and survival in transitional cell carcinoma of the upper urinary tract: A 30-year experience in 252 patients. Urology 1998, 52, 594-601. [CrossRef]

7. Ito, K.; Kuroda, K.; Asakuma, J.; Hamada, S.; Tachi, K.; Tasaki, S.; Sato, A.; Horiguchi, A.; Seguchi, K.; Asano, T. Preoperative risk factors for extraurothelial recurrence in patients with ureteral cancer treated with radical nephroureterectomy. J. Urol. 2014, 191, 1685-1692. [CrossRef]

8. Kondo, T.; Nakazawa, H.; Ito, F.; Hashimoto, Y.; Toma, H.; Tanabe, K. Impact of the extent of regional lymphadenectomy on the survival of patients with urothelial carcinoma of the upper urinary tract. J. Urol. 2007, 178, 1212-1217. [CrossRef] [PubMed]

9. Kondo, T.; Tanabe, K. The role of lymph node dissection in the management of urothelial carcinoma of the upper urinary tract. Int. J. Clin. Oncol. 2011, 16, 170-178. [CrossRef] [PubMed]

10. Roscigno, M.; Cozzarini, C.; Bertini, R.; Scattoni, V.; Freschi, M.; Da Pozzo, L.F.; Briganti, A.; Gallina, A.; Capitanio, U.; Colombo, $\mathrm{R}$. Prognostic value of lymph node dissection in patients with muscle-invasive transitional cell carcinoma of the upper urinary tract. Eur. Urol. 2008, 53, 794-802. [CrossRef]

11. Raman, J.D.; Lin, Y.K.; Kaag, M.; Atkinson, T.; Crispen, P.; Wille, M.; Smith, N.; Hockenberry, M.; Guzzo, T.; Peyronnet, B.; et al. High rates of advanced disease, complications, and decline of renal function after radical nephroureterectomy. Urol. Oncol. 2014, 32, 47.e9-47.e14. [CrossRef]

12. Saito, K.; Kawakami, S.; Ohtsuka, Y.; Fujii, Y.; Masuda, H.; Kumagai, J.; Kobayashi, T.; Kageyama, Y.; Kihara, K. The impact of preoperative serum $\mathrm{C}$-reactive protein on the prognosis of patients with upper urinary tract urothelial carcinoma treated surgically. BJU Int. 2007, 100, 269-273. [CrossRef]

13. Kikuchi, E.; Margulis, V.; Karakiewicz, P.I.; Roscigno, M.; Mikami, S.; Lotan, Y.; Remzi, M.; Bolenz, C.; Langner, C.; Weizer, A. Lymphovascular invasion predicts clinical outcomes in patients with node-negative upper tract urothelial carcinoma. J. Clin. Oncol. 2009, 27, 612-618. [CrossRef]

14. Margulis, V.; Shariat, S.F.; Matin, S.F.; Kamat, A.M.; Zigeuner, R.; Kikuchi, E.; Lotan, Y.; Weizer, A.; Raman, J.D.; Wood, C.G. Outcomes of radical nephroureterectomy: A series from the Upper Tract Urothelial Carcinoma Collaboration. Cancer 2009, 115, 1224-1233. [CrossRef]

15. Zigeuner, R.; Shariat, S.F.; Margulis, V.; Karakiewicz, P.I.; Roscigno, M.; Weizer, A.; Kikuchi, E.; Remzi, M.; Raman, J.D.; Bolenz, C. Tumour necrosis is an indicator of aggressive biology in patients with urothelial carcinoma of the upper urinary tract. Eur. Urol. 2010, 57, 575-581. [CrossRef] [PubMed]

16. Rouprêt, M.; Babjuk, M.; Compérat, E.; Zigeuner, R.; Sylvester, R.J.; Burger, M.; Cowan, N.C.; Böhle, A.; Van Rhijn, B.W.; Kaasinen, E.; et al. European Association of Urology Guidelines on Upper Urinary Tract Urothelial Cell Carcinoma: 2015 Update. Eur. Urol. 2015, 68, 868-879. [CrossRef]

17. Favaretto, R.L.; Shariat, S.F.; Chade, D.C.; Godoy, G.; Adamy, A.; Kaag, M.; Bochner, B.H.; Coleman, J.; Dalbagni, G. The effect of tumor location on prognosis in patients treated with radical nephroureterectomy at Memorial Sloan-Kettering Cancer Center. Eur. Urol. 2010, 58, 574-580. [CrossRef]

18. Otto, W.; Shariat, S.F.; Fritsche, H.M.; Gupta, A.; Matsumoto, K.; Kassouf, W.; Martignoni, G.; Walton, T.J.; Tritschler, S.; Baba, S.; et al. Concomitant carcinoma in situ as an independent prognostic parameter for recurrence and survival in upper tract urothelial carcinoma: A multicenter analysis of 772 patients. World J. Urol. 2011, 29, 487-494. [CrossRef] [PubMed]

19. Cho, K.S.; Hong, S.J.; Cho, N.H.; Choi, Y.D. Grade of hydronephrosis and tumor diameter as preoperative prognostic factors in ureteral transitional cell carcinoma. Urology 2007, 70, 662-666. [CrossRef]

20. Rink, M.; Robinson, B.D.; Green, D.A.; Cha, E.K.; Hansen, J.; Comploj, E.; Margulis, V.; Raman, J.D.; Ng, C.K.; Remzi, M. Impact of histological variants on clinical outcomes of patients with upper urinary tract urothelial carcinoma. J. Urol. 2012, 188, 398-404. [CrossRef] [PubMed]

21. Li, W.-M.; Li, C.-C.; Ke, H.-L.; Wu, W.-J.; Huang, C.-N.; Huang, C.-H. The prognostic predictors of primary ureteral transitional cell carcinoma after radical nephroureterectomy. J. Urol. 2009, 182, 451-458. [CrossRef]

22. Kuroda, K.; Asakuma, J.; Horiguchi, A.; Kawaguchi, M.; Shinchi, M.; Masunaga, A.; Tasaki, S.; Sato, A.; Ito, K. Chronic kidney disease and positive surgical margins as prognosticators for upper urinary tract urothelial carcinoma patients undergoing radical nephroureterectomy. Mol. Clin. Oncol. 2019, 10, 547-554. [CrossRef]

23. Stewart, J.H.; Buccianti, G.; Agodoa, L.; Gellert, R.; McCredie, M.R.; Lowenfels, A.B.; Disney, A.P.; Wolfe, R.A.; Boyle, P.; Maisonneuve, P. Cancers of the kidney and urinary tract in patients on dialysis for end-stage renal disease: Analysis of data from the United States, Europe, and Australia and New Zealand. J. Am. Soc. Nephrol. 2003, 14, 197-207. [CrossRef]

24. Tonelli, M.; Wiebe, N.; Culleton, B.; House, A.; Rabbat, C.; Fok, M.; McAlister, F.; Garg, A.X. Chronic kidney disease and mortality risk: A systematic review. J. Am. Soc. Nephrol. 2006, 17, 2034-2047. [CrossRef] 
25. Fried, L.F.; Katz, R.; Sarnak, M.J.; Shlipak, M.G.; Chaves, P.H.; Jenny, N.S.; Stehman-Breen, C.; Gillen, D.; Bleyer, A.J.; Hirsch, C. Kidney function as a predictor of noncardiovascular mortality. J. Am. Soc. Nephrol. 2005, 16, 3728-3735. [CrossRef]

26. Liberati, A.; Altman, D.G.; Tetzlaff, J.; Mulrow, C.; Gøtzsche, P.C.; Ioannidis, J.P.; Clarke, M.; Devereaux, P.J.; Kleijnen, J.; Moher, D. The PRISMA statement for reporting systematic reviews and meta-analyses of studies that evaluate health care interventions: Explanation and elaboration. J. Clin. Epidemiol. 2009, 62, e1-e34. [CrossRef]

27. Yu, H.S.; Hwang, J.E.; Chung, H.S.; Cho, Y.H.; Kim, M.S.; Hwang, E.C.; Oh, K.J.; Kim, S.-O.; Jung, S.I.; Kang, T.W. Is preoperative chronic kidney disease status associated with oncologic outcomes in upper urinary tract urothelial carcinoma? A multicenter propensity score-matched analysis. Oncotarget 2017, 8, 66540-66549. [CrossRef]

28. Xylinas, E.; Rink, M.; Margulis, V.; Clozel, T.; Lee, R.K.; Comploj, E.; Novara, G.; Raman, J.D.; Lotan, Y.; Weizer, A.; et al. Impact of renal function on eligibility for chemotherapy and survival in patients who have undergone radical nephro-ureterectomy. $B J U$ Int. 2013, 112, 453-461. [CrossRef] [PubMed]

29. Momota, M.; Hatakeyama, S.; Tokui, N.; Sato, T.; Yamamoto, H.; Tobisawa, Y.; Yoneyama, T.; Yoneyama, T.; Hashimoto, Y.; Koie, T.; et al. The Impact of Preoperative Severe Renal Insufficiency on Poor Postsurgical Oncological Prognosis in Patients with Urothelial Carcinoma. Eur. Urol. Focus 2019, 5, 1066-1073. [CrossRef] [PubMed]

30. Jan, H.C.; Yang, W.H.; Ou, C.H. Combination of the Preoperative Systemic Immune-Inflammation Index and MonocyteLymphocyte Ratio as a Novel Prognostic Factor in Patients with Upper-Tract Urothelial Carcinoma. Ann. Surg. Oncol. 2019, 26, 669-684. [CrossRef] [PubMed]

31. Freifeld, Y.; Ghandour, R.; Singla, N.; Woldu, S.; Clinton, T.; Kulangara, R.; Bagrodia, A.; Matin, S.F.; Petros, F.G.; Raman, J.D.; et al. Preoperative predictive model and nomogram for disease recurrence following radical nephroureterectomy for high grade upper tract urothelial carcinoma. Urol. Oncol. 2019, 37, 758-764. [CrossRef]

32. Koguchi, D.; Matsumoto, K.; Ikeda, M.; Taoka, Y.; Hirayama, T.; Murakami, Y.; Utsunomiya, T.; Matsuda, D.; Okuno, N.; Irie, A.; et al. Investigation of estimated glomerular filtration rate and its perioperative change in patients with upper urinary tract urothelial carcinoma: A multi-institutional retrospective study. Asia Pac. J. Clin. Oncol. 2018, 14, e420-e427. [CrossRef]

33. Xing, Y.; Xiong, G.; Fang, D.; Yang, X.; Li, X.; Zhou, L. Prognostic Value of Gene Methylation and Clinical Factors in Non-MuscleInvasive Upper Tract Urothelial Carcinoma After Radical Nephroureterectomy. Clin. Genitourin. Cancer 2016, 14, e371-e378. [CrossRef]

34. Huang, Y.C.; Chang, Y.H.; Chiu, K.H.; Shindel, A.W.; Lai, C.H. Adjuvant radiotherapy for locally advanced upper tract urothelial carcinoma. Sci. Rep. 2016, 6, 38175. [CrossRef]

35. Yeh, H.C.; Jan, H.C.; Wu, W.J.; Li, C.C.; Li, W.M.; Ke, H.L.; Huang, S.P.; Liu, C.C.; Lee, Y.C.; Yang, S.F.; et al. Concurrent Preoperative Presence of Hydronephrosis and Flank Pain Independently Predicts Worse Outcome of Upper Tract Urothelial Carcinoma. PLoS ONE 2015, 10, e0139624. [CrossRef]

36. Morizane, S.; Yumioka, T.; Yamaguchi, N.; Masago, T.; Honda, M.; Sejima, T.; Takenaka, A. Risk stratification model, including preoperative serum C-reactive protein and estimated glomerular filtration rate levels, in patients with upper urinary tract urothelial carcinoma undergoing radical nephroureterectomy. Int. Urol. Nephrol. 2015, 47, 1335-1341. [CrossRef] [PubMed]

37. Higgins, J.P.; Thompson, S.G.; Deeks, J.J.; Altman, D.G. Measuring inconsistency in meta-analyses. BMJ 2003, 327, 557-560. [CrossRef] [PubMed]

38. Ehdaie, B.; Shariat, S.F.; Savage, C.; Coleman, J.; Dalbagni, G. Postoperative nomogram for disease recurrence and cancer-specific death for upper tract urothelial carcinoma: Comparison to American Joint Committee on Cancer staging classification. Urol. J. 2014, 11, 1435-1441. [PubMed]

39. Lee, K.-H.; Chen, Y.-T.; Chung, H.-J.; Liu, J.-S.; Hsu, C.-C.; Tarng, D.-C. Kidney disease progression in patients of upper tract urothelial carcinoma following unilateral radical nephroureterectomy. Ren. Fail. 2016, 38, 77-83. [CrossRef]

40. Leppert, J.T.; Lamberts, R.W.; Thomas, I.-C.; Chung, B.I.; Sonn, G.A.; Skinner, E.C.; Wagner, T.H.; Chertow, G.M.; Brooks, J.D. Incident CKD after radical or partial nephrectomy. J. Am. Soc. Nephrol. 2018, 29, 207-216. [CrossRef]

41. Clark, M.A.; Shikanov, S.; Raman, J.D.; Smith, B.; Kaag, M.; Russo, P.; Wheat, J.C.; Wolf, J.S.; Matin, S.F.; Huang, W.C. Chronic kidney disease before and after partial nephrectomy. J. Urol. 2011, 185, 43-48. [CrossRef]

42. Yoneyama, T.; Tobisawa, Y.; Yoneyama, T.; Yamamoto, H.; Imai, A.; Mori, K.; Hatakeyama, S.; Hashimoto, Y.; Koie, T.; Ohyama, C. Sequential chemotherapy with gemcitabine plus carboplatin, followed by additional docetaxel for aged patients with advanced upper-tract urothelial cancer. J. Clin. Oncol. 2015, 33, 344. [CrossRef]

43. Birtle, A.; Johnson, M.; Chester, J.; Jones, R.; Dolling, D.; Bryan, R.T.; Harris, C.; Winterbottom, A.; Blacker, A.; Catto, J.W. Adjuvant chemotherapy in upper tract urothelial carcinoma (the POUT trial): A phase 3, open-label, randomised controlled trial. Lancet 2020, 395, 1268-1277. [CrossRef]

44. Von Hoff, D.D.; Schilsky, R.; Reichert, C.M.; Reddick, R.L.; Rozencweig, M.; Young, R.C.; Muggia, F.M. Toxic effects of cisdichlorodiammineplatinum (II) in man. Cancer Treat. Rep. 1979, 63, 1527-1531. [PubMed]

45. Arany, I.; Safirstein, R.L. Cisplatin nephrotoxicity. Semin. Nephrol. 2003, 23, 460-464. [CrossRef]

46. Pabla, N.; Dong, Z. Cisplatin nephrotoxicity: Mechanisms and renoprotective strategies. Kidney Int. 2008, 73, 994-1007. [CrossRef] 